\title{
Analysis Situs and Image Processing
}

\author{
Fridrich Sloboda and Bedrich Zatko
}

\begin{abstract}
Institute of Control Theory and Robotics, Slovak Academy of Sciences, Dúbravska cesta 9,84237 Bratislava, Slovakia
\end{abstract}

\begin{abstract}
In the paper a topological approach to approximation of planar Jordan curves and arcs is described. The approximation is based on the basic notions of intrinsic geometry of metric spaces: on the notion of a shortest path in a polygonaly bounded compact set and on the notion of a geodesic diameter of a polygon. Furthermore, a new linear time algorithm for the shortest path problem solution is described, and the approximation of the most important characteristic set in image processing is shown.
\end{abstract}

\section{Introduction}

Analysis situs, i.e. topology, is a part of geometry related to investigation of invariants of connected compact sets. In two-dimensional case the boundaries of connected compact sets represent planar curves. The notion of a curve belongs to the hardest problems in the history of mathematics. The modern history is related to C. Jordan. A planar Jordan curve is a simple closed curve, that is a curve which belongs to a parametrized path $\phi:[a, b] \rightarrow R^{2}$ with $a \neq b$, $\phi(a)=\phi(b), \phi(s) \neq \phi(t)$ for all $a \leq s<t<b$. Though the class of planar curves which possess parametric forms is large, not all planar curves can be expressed in this form. Therefore there was an interest to find a more general definition of a curve. The first topological definition of a planar curve was given by $G$. Cantor : a planar curve is a connected compact set of points, which does not possess internal points [11]. The most general topological definition was introduced by P. Urysohn and K. Menger : a curve is an one-dimensional connected compact set, whereby a connected compact set $S \subset R^{n}$ is one-dimensional, if $\forall s \in S \exists \bar{\delta}=$ $\bar{\delta}(s)>0: \forall \delta \leq \bar{\delta}:$

$$
M(s, \delta)=\left\{x \in R^{n} \mid \operatorname{dist}(x, s)=\delta\right\} \cap S
$$

does not possess a connected compact component, which consists of more than one point $[9,11,17]$. It has been shown that in the case of planar curves both topological definitions are equivalent [11]. A connected compact set $S \subset R^{2}$ is a simple closed Urysohn curve if $\forall s \in S \exists \bar{\delta}=\bar{\delta}(s)>0: \forall \delta \leq \bar{\delta}: M(s, \delta)$ defined by (1) possesses exactly two connected compact components, which consist of one point. A connected compact set $S \subset R^{2}$ is a simple Urysohn arc, if $\forall s \in S \backslash$ $\left\{s_{1}, s_{2}\right\} \exists \bar{\delta}=\bar{\delta}(s)>0 ; \forall \delta \leq \bar{\delta}: M(s, \delta)$ defined by (1) possesses exactly two connected compact components, which consist of one point, and $\forall s \in\left\{s_{1}, s_{2}\right\} \in S$ 
$\exists \bar{\delta}=\bar{\delta}(s)>0: \forall \delta \leq \bar{\delta}: M(s, \delta)$ defined by (1) possesses exactly one connected compact component, which consists of one point. Not all planar Cantor (Urysohn) curves possess a parametric form. A planar Cantor (Urysohn) curve is a continuous image of a linear segment iff it is locally connected $[9,11]$. The notion of local connectedness was introduced by S. Mazurkiewicz [8] and H. Hahn [5]. A set is locally connected if each point of the set possesses an arbitrary small connected neighborhood. A simple planar Urysohn curve (a simple planar Urysohn arc) is locally connected, so that it is parametrizable $[9,11]$. But if a curve is parametrizable it does not imply that the parametric form itself will be ever found. W. Sierpinski has shown an example of a planar curve, so called Sierpinski carpet, which has a unique property: it contains all planar curves. To each planar Cantor (Urysohn) curve $C$ there exists a subset $C$ of the Sierpinski carpet which is homeomorph with $C$ [11].

The trace of one-dimensional continua which do not possess a parametric form or if this form is not available is "untouchable". Untouchable in the sense, that it is not possible to generate points which lie on the trace of such continua. How to measure the length of untouchable one-dimensional continua? How to represent such continua? These questions are related to the basic problems of set theoretical topology, but they are related also to the basic problems of image processing.

\section{Inner and Outer Jordan Content}

In order to answer above mentioned questions let us consider a regular grid in $R^{2}$. The grid itself represents a theoretical tool of set--theoretical topology and was introduced by C. Jordan and G. Peano by the end of last century in order to define measurable sets. More formally, for $p=0,1,2, \ldots$ and for each couple $\left(w_{1}, w_{2}\right)$ of integer numbers let

$$
N_{\left(w_{1}, w_{2}\right)}^{p}=\left\{x \in R^{2} \mid w_{i} 2^{-p} \leq x_{i} \leq\left(w_{i}+1\right) 2^{-p}, i=1,2\right\} .
$$

$N_{\left(w_{1}, w_{2}\right)}^{p}$ represents the topological unit of an orthogonal grid.

Let $M \subset R^{2}$ be a simply connected compact set, and let us define

$$
I_{p}^{+}(M)=\sum I\left(N_{\left(w_{1}, w_{2}\right)}^{p}\right), \quad I_{p}^{-}(M)=\sum I\left(N_{\left(w_{1}, w_{2}\right.}^{p}\right),
$$

where $I$ denotes the content of (.), and the sum defining $I_{p}^{+}$is taken over all squares for which $N_{\left(w_{1}, w_{2}\right)}^{p} \cap M \neq \emptyset$ and $I_{p}^{-}$corresponds to squares for which $N_{\left(w_{1}, w_{2}\right)}^{p} \subset M^{o} . M$ is measurable if

$$
I(M)=\inf _{p} I_{p}^{+}(M)=\sup _{p} I_{p}^{-}(M)=\lim _{p \rightarrow \infty} I_{p}^{ \pm}(M),
$$

where $I(M)$ is the Jordan content of $M . M$ is measurable iff the boundary of $M$, $\partial M$, has measure zero. A set which has measure zero does not possess internal points, i.e., it does not possess a square $N_{\left(w_{1}, w_{2}\right)}^{p}$ internal to this set. 
Squares with $N_{\left(w_{1}, w_{2}\right)}^{p} \subset M^{\circ}$ will be called inner elements of $M$ and squares with $N_{\left(w_{1}, w_{2}\right)}^{p} \cap M \neq \emptyset$ boundary elements of $M$. Let

$$
{ }^{+} M_{p}=\bigcup_{I_{p}^{+}} N_{\left(w_{1}, w_{2}\right)}^{p},-M_{p}=\bigcup_{I_{p}^{+}} N_{\left(w_{1}, w_{2}\right)}^{p},
$$

where ${ }^{+} M_{p},-M_{p}$, corresponds to those $N_{\left(w_{1}, w_{z}\right)}^{p}$ which belong to $I_{p}^{+}, I_{p}^{-}$, respectively. Note that there exists $p$ such that ${ }^{+} M_{p}$ and $-M_{p}$ are simply connected. Further, ${ }^{+} M_{p}\left(-M_{p}\right)$ will be called edge connected if each element of ${ }^{+} M_{p}\left({ }^{-} M_{p}\right)$ shares a common edge with some other element of ${ }^{+} M_{p}\left(-M_{p}\right)$.

The approximation of a smooth planar Jordan curves and arcs is given by the following $[12,13]$

Theorem 1: Let $\gamma:[0, d(\gamma)] \rightarrow R^{2}$ be a smooth Jordan curve with bounded length $d(\gamma)$. Let $G_{p}=+M_{p} \backslash-M_{p}^{\circ}$ be edge connected, $p=0,1, \ldots$ Let $p_{p}$ : $\left[0, d\left(p_{p}\right)\right] \rightarrow R^{2}$ denote the shortest Jordan curve in $G_{p}$ containing $-M_{p}, p=$ $0,1, \ldots$ Then

$$
\lim _{k} d\left(p_{k}\right)=d(\gamma)
$$

Theorem 2: Let $\gamma:[0, d(\gamma)] \rightarrow R^{2}$ be a smooth Jordan arc with bounded length $d(\gamma)$. Let

$$
G_{p}=\cup N_{\left(w_{1}, w_{2}\right)}^{p},
$$

where $G_{p}$ corresponds to squares for which $N_{\left(w_{1}, w_{2}\right)}^{p} \cap M \neq \emptyset$. Let $G_{p}$ be edge connected, $p=0,1, \ldots$, and let $g_{p}:\left[0, d\left(g_{p}\right)\right] \rightarrow R^{2}$ be a geodesic diameter corresponding to $G_{p}, p=0,1, \ldots$. Then

$$
\lim _{p} d\left(g_{p}\right)=d(\gamma)
$$

According to these theorems the length of a planar Jordan curve and the length of a planar Jordan arc is defined on basis of the basic notions of intrinsic geometry of metric spaces [1]: on the notion of the shortest Jordan curve in a polygonally bounded compact set and on the notion of a geodesic diameter in a polygon. The approximating curves are represented by grid points, i.e., by points whose coordinates are integer numbers. The achievements in high technology have allowed to produce high resolution monitors, plotters, scanners and CCD cameras. All of them are built on the basis of an orthogonal grid. The resolution has been increased up to 2 microns at the present linear scanners. The achievements in high technology have set up new demands on algorithms and their complexity. They have created new scientific directions, such as computer aided geometric design, computer graphics and image processing. 


\section{Implicit Forms and Characteristic Sets}

In the previous section theoretical gridding technique was considered. The practical gridding is related to the approximation of planar Jordan curves and arcs in implicit forms [15]:

Theorem 3: Let $D \subset R^{2}$ be connected and compact, and let $f: D \subset R^{2} \rightarrow R^{1}$ be a continuously differentiable function: $\exists x^{*} \in D^{0}: \forall x \neq x^{*} \in D: f(x)<f\left(x^{*}\right)$, where $f\left(x^{*}\right)$ is the only local maximum of $f$ in $D$. Let

$$
L(z)=\{x \in D \mid f(x)-z=0\}
$$

be a simple planar Urysohn curve. Let $p_{p}:\left[0, d\left(p_{p}\right)\right] \rightarrow R^{2}$ be the noncontractible shortest Jordan curve in

$$
G_{p}^{\prime}=\cup N_{\left(w_{1}, w_{2}\right)}^{p}
$$

where $N_{\left(w_{1}, w_{2}\right)}^{p}$ are squares, which have the property that not all of their vertices have the same value of $\operatorname{sign}[f(x)-z=0]$, and $\partial G_{p}^{\prime}=L_{1}^{(p)} \cup L_{2}^{(p)}, L_{1}^{(p)} \subset I\left(L_{2}^{(p)}\right)$. Then

$$
\lim d\left(p_{p}\right)=d(L)
$$

where $d(L)$ is the length of $L(z)$.

Proof. According to the assumption

$$
L(z)=\{x \in D \mid f(x)-z=0\}
$$

is a simple planar Urysohn curve. Any simple planar Urysohn curve is locally connected, so that it is parametrizable, and it is homeomorph with the unit circle. Because $f$ is continuously differentiable $L(z)$ is rectifiable.

Let us consider the orthogonal grid defined by (2). Because $f: R^{2} \rightarrow R^{1}$ is continuously differentiable, there exists $p$ such that $G_{p}^{\prime}$ consists of squares, which have the property that $L(z)$ enters and leaves a square exactly once. According to the definition $G_{p}^{\prime}$ consists of squares, which have the property that not all of their vertices have the same value of $\operatorname{sign}[f(x)-z=0]$. But for any grid points resolution there might exist squares, which were entered and left by the trace of $L(z)$ through the same edge. These squares do not belong to $G_{p}^{t}$ and can not be identified. Let $p_{p}:\left[0, d_{p}\right] \rightarrow R^{2}$ denote the shortest Jordan curve in $G_{p}^{\prime}$, encircling $L_{1}^{(p)}$. Because $f: R^{2} \rightarrow R^{1}$ is continuously differentiable, there exists $p$ such that the squares which were entered and left by the trace of $L(z)$ through the same edge, share exactly one edge with a square belonging to $G_{p}^{\prime}$. In this situation the shortest polygonal path in $G_{p}^{\prime}$ can not pass through such squares, otherwise it can be shortened. According to this the shortest polygonal path in $G_{p}^{\prime}$ is identical to the shortest polygonal path in $G_{p}$, so that Theorem 1 applies.

A similar theorem holds for length approximation of smooth planar Jordan arcs in implicit forms [15]. 
Comment: The consequence of Theorem 3 is, that if the inner content elements are given, for the approximation of the boundary it is sufficient to border the inner content elements by elements which share one edge or one vertex with an inner content element, and in this set to find the shortest Jordan curve.

Let $f: D \subset R^{2} \rightarrow R^{1}$ be twice continuously differentiable. Surface analysis of $f$ is related to approximation and representation of the following planar curves and arcs in implicit forms

$$
\begin{gathered}
L(z)=\{x \in D \mid f(x)-z=0\} \\
\text { det } H=0, \text { for }(g, g) \neq 0,
\end{gathered}
$$

where $H$ is the Hessian matrix of $f$ and $g$ is the gradient vector of $f$.

(6) represents equiconstant level sets of $f$, and enables to visualize $f$. (7) represents the boundary of convexity (pseudoconvexity), or concavity (pseudoconcavity). Let $y \in R^{2}$ be a vector such that $\|y\|=1$. Then $\partial f / \partial y=(g, y)$, where $g$ is the gradient vector of $f$ and $\partial^{2} f / \partial y^{2}=(H y, y)$, where $H$ is the Hessian matrix of $f[10]$. If $y=g^{N}$, where $g^{N}$ is the normalized gradient vector, then $(g, y)=\|g\|\|y\| \cos (g, y)=\|g\|$. Suppose that for a given $f: D \subset R^{2} \rightarrow R^{1}$ there is $\epsilon>0$, such that the set

$$
L^{*}=\left\{x \in D \mid \max _{-\epsilon<\alpha<\epsilon}(g(x+\alpha y), y)=(g(x), y)\right\}
$$

is nonempty. The first directional derivative possesses its maximum where the second directional derivative vanishes, which corresponds to the set

$$
L^{*}=\left\{x \in D \subset R^{2} \mid(H y, y)=0\right\} .
$$

Because $\|y\|=1,(H y, y)=0$ if and only if $\operatorname{det} H=0 . f: R^{2} \rightarrow R^{1}$ is convex on a convex set $D \subset R^{2}$ if and only if the Hessian matrix is positive semidefinite. Moreover, $f$ is strictly convex on $D$ if $H$ is positive definite [10]. A $2 \times 2$ Hessian matrix is positive definite if $\partial^{2} f / \partial x_{1}^{2}>0$ and det $H>0$ and is negative definite if $\partial^{2} f / \partial x_{1}^{2}<0$ and det $H>0$. It means that the boundary of a characteristic set on which $f$ is convex or concave, respectively, is related to the set $L^{*}=\left\{x \in D \subset R^{2} \mid \operatorname{det} H=0\right\}$. In the case, the boundary of a compact set, where $\operatorname{det} H=0$ is not convex, we speak about pseudoconvexity, pseudoconcavity, respectively.

Comment: In image processing, the set where the norm of the gradient of $f$ : $R^{2} \rightarrow R^{1}$ is locally maximal, is related to the set where the Laplace operator vanishes [7]. The Laplace operator is the trace of the Hessian matrix of $f$, and is by no way related to the set where the norm of the gradient vector is locally maximal. The set where the norm of the gradient vector is locally maximal is related to the set where $\operatorname{det} H=0$, which is well known in the theory of convexity, and this set is the most important set according to which a segmentation of $f: R^{2} \rightarrow R^{1}$ can be performed $[4,10,18,19]$. In some cases this set represents an equiconstant level set of $f$. 


\section{$4 \quad$ Algorithm}

Let $G=P_{L_{2}} \backslash P_{L_{1}}^{o}$, where $P_{L_{1}}, P_{L_{2}}$ are polygons, $P_{L_{1}} \subset P_{L_{2}}^{o}$. The vertices of the shortest polygonal path in $G$ have the following property [14]:

Theorem 4: Let $P_{L_{1}}, P_{L_{2}}$, be polygons: $P_{L_{1}} \subset P_{L_{2}}^{\circ}, \partial P_{L_{1}}=L_{1}, \partial P_{L_{2}}=L_{2}$. A vertex of $L_{1}, L_{2}$, is a vertex of the shortest polygonal curve in $G=P_{L_{2}} \backslash P_{L_{1}}^{o}$ encircling $L_{1}$ iff there exists a linear segment $S$ passing through this vertex, endpoints of which belong to $L_{2}, L_{1}$, respectively.

The linear segments related to Theorem 4 are called relative support lines, and represent a generalization of the notion of a support line introduced by $\mathrm{G}$. Minkowski. The shortest polygonal path itself has the property [14]:

Theorem 5: Let $p_{0}, p_{1}, \ldots p_{n-1}, p_{0} \equiv p_{n-1}$ be a polygonal curve in $G=$ $P_{L_{2}} \backslash P_{L_{1}}^{o}, P_{L_{1}} \subset P_{L_{2}}^{o}, \partial P_{L_{1}}=L_{1}, \partial P_{L_{2}}=L_{2}$, encircling $L_{1}$ in the positive sense. Let $p_{0}$ be a vertex of the shortest polygonal curve in $G$. Then $p_{0}, p_{1}, \ldots, p_{n-1}$ is a shortest polygonal curve in $G$ encircling $L_{1}$ iff $\vec{p}_{i} \vec{p}_{i+1(\bmod n)}$ points on $L_{2}, L_{1}$ if $p_{i+1(\bmod n)} \in L_{1}, p_{i+1(\bmod n)} \in L_{2}$, respectively.

Almost all algorithms for the shortest path problem solution are based on partition of $G[3,6]$. The most popular partition of $G$ is the triangulation of $G$ [2]. According to Theorem 4 the pseudomonotone polygon partition of $G$ is related only to extremal vertices of $L_{1}, L_{2}$, respectively, which is the smallest subset of all vertices of $G$ according to which a partition of $G$ can be performed.

The algorithm for the shortest path problem solution in $G$, related to image processing problems, is shown on Fig. 1 and described as follows [14]:

\section{Algorithm}

Step (1): Find all extremal convex vertices of $P_{L_{1}}$ and all extremal concave vertices of $P_{L_{2}}$.

Step (2): Find all x-axis parallel pseudodiagonals of $G$ related to extremal convex vertices of $P_{L_{1}}$ and extremal concave vertices of $P_{L_{2}}$.

Step (3): Eliminate all monotone polygons whose boundaries contain only vertices of $L_{1}, L_{2}$, respectively; and denote the resulting polygonally bounded set by $G^{\prime}$.

Step (4): Find a shortest path in each pseudomonotone polygon between two extremal vertices of $G^{\prime}$.
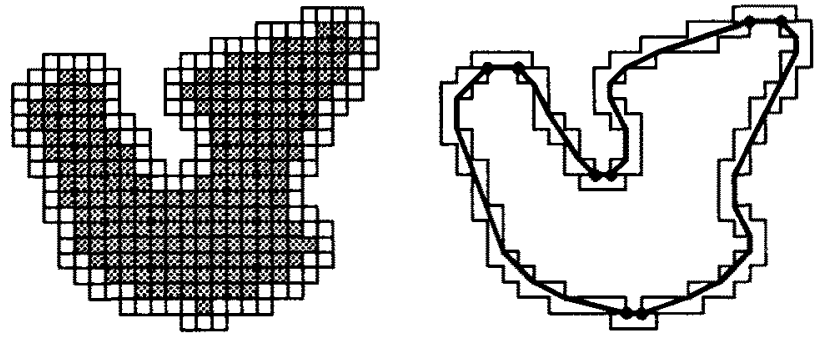

Fig. 1. 
Triangulation and pseudomonotone polygon partition of $G$ are based on trapezoidation, which can be performed in linear time [2]. In view of Theorem 5 , Step (4) has linear time complexity which implies that Algorithm has also linear time complexity.

Comment: In image processing trapezoidation does not require a special procedure, because it is a part of the connectivity analysis. In this case implicit forms are related to $f: R^{2} \rightarrow R^{1}$ which is given by discrete function values.

Geodesic diameter calculation has $O(n \log n)$ time complexity, where $n$ is the number of the polygon [16].

\section{Examples}

Let us consider the following (see Fig. 2)

Example: $f(x, y)=\exp \left(-\frac{1}{30}(x-2)^{2}-\frac{1}{20}(x-2)(y-2)-\frac{1}{20}(y-2)^{2}\right)-$ $-\exp \left(-\frac{1}{40} x^{2}-\frac{1}{20}(y+2)^{2}\right)+\exp \left(-\frac{1}{20}(x+4)^{2}-\frac{1}{20}(y-2)^{2}\right)$.

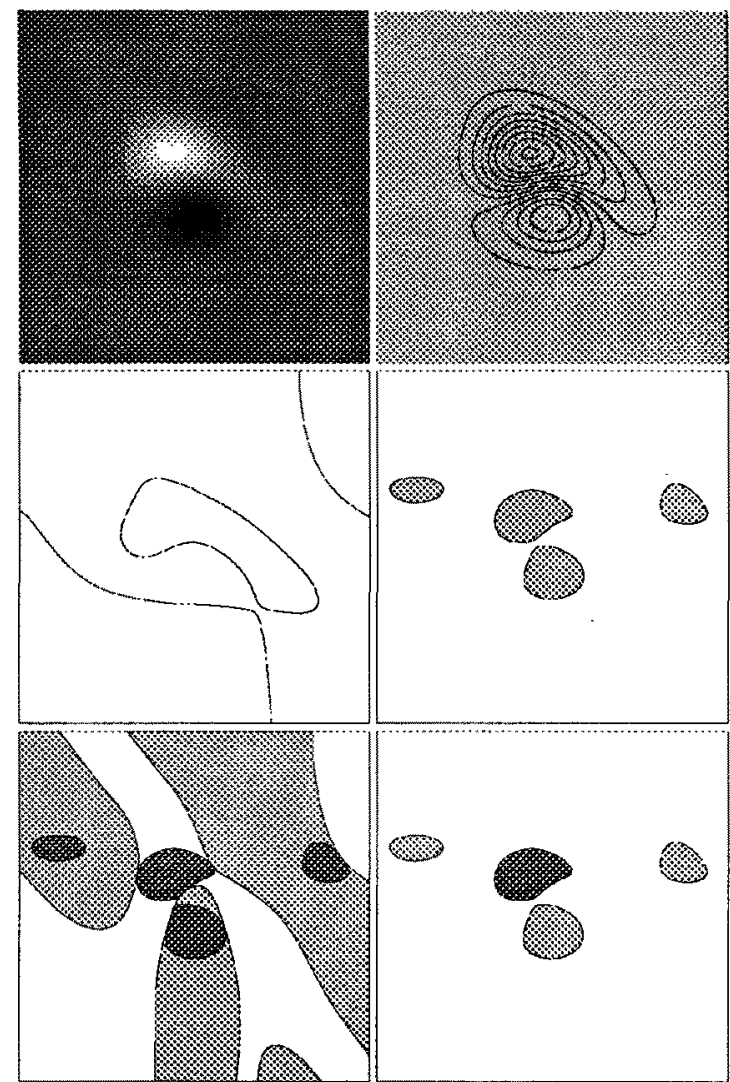

Fig. 2. 
Fig. 2 shows in the first rows the function values of $f$ and the corresponding equiconstant level sets of $f$. In the second rows are shown sets where Laplacian operator vanishes and $\operatorname{det} H \geq 0$, and in the third rows are shown sets where the second partial derivative $\partial f / \partial x^{2}$ is positive or negative, and sets where $f$ is concave (pseudoconcave) or convex (pseudoconvex).

\section{References}

1. A.D. Alexandrov, Intrinsic Geometry of Convex Surfaces, (in Russian) OGIZ, Moscow (1948),

2. B. Chazelle, Triangulating a Simple Polygon in Linear Time, Discrete Comput. Geom. 6 (1991) 485-524.

3. B. Chazelle, A theorem on polygon cutting with applications, Proc. 23-rd Annual Symp, on Found. Comput. Sci. (1982) 339-349.

4. H. Enomoto, T. Katayama, Structure Lines of Images, Proc. of 3rd Int. Joint Conf. On Pattern Recognition, (1976) 811-815.

5. H. Hahn, Über die Komponenten offener Mengen, Fundamenta Math. (1921) 189192.

6. D. T. Lee, F. P. Preparata, Euclidean Shortest Paths in the Presence of Rectilinear Barriers, Networks 14 (1984) 393-410.

7. D. Marr, E.C. Hildreth, Theory of Edge Detection, Proc. Rog. Soc. London (1980) $187-217$.

8. S. Mazurkiewicz, Sur les lignes de Jordan, Fundamenta Math. (1920) 166-209.

9. K. Menger, Kurventheorie, B.G. Teubner Press, Leipzig, (1932).

10. J.M. Ortega, W.C. Rheinboldt, Iterative Solution of Nonlinear Equation in Several Variables, Academic Press, New York, London (1970).

11. A.S.Parchomenko, Was ist eine Kurve, VEB Deutscher Verlag der Wissenschaften, Berlin (1957) (original Moscow (1954)).

12. F.Sloboda, J.Stoer, On Piecewiese Linear Approximation of Planar Jordan Curves, J. Comp. Applied Mathematics 55 (1994) 369-383.

13. F. Sloboda, J. Stoer, On Piecewise Linear Approximation of Planar Jordan Arcs., Tech. Report of the Inst. of Control Theory and Robotics, Bratislava (1996).

14. F. Sloboda, B. Zatko, On Linear Time Algorithm for the Shortest Path Problem in a Polygonally Bounded Compact Set, Techn. Report of the Inst. of Control Theory and Robotics, Bratislava (1996).

15. F. Sloboda, B. Zatko, On Approximation of Planar Jordan Curves and Arcs in Implicit Forms, Techn. Report of the Inst. of Control Theory and Robotics, Bratislava (1997).

16. S. Suri, The All Geodesic-Further Neighbours Problem for Simple Polygons, Proc. Third Ann. Symp. on Comp. Geom. (1987) 64-75.

17. P. Urysohn, Mémoire sur les multiplicités Cantoriennes, Fundamenta Math. (1925), 30-130.

18. A. H. Wallace, Differential Topology, N. Y., W. A. Benjamin, (1968).

19. Y. Watanabe, Structural Features of Three-Dimensional Images, Proc. 1st Int. Symp. for Science on Form, KTK Scientific Publ. Tokyo, (1986) 247-254. 